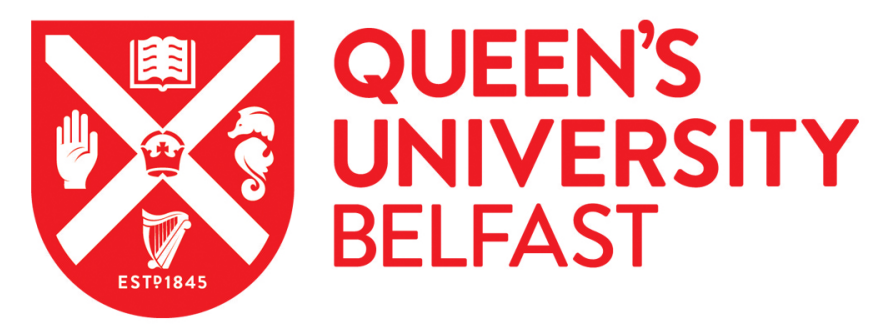

\title{
Network Topology, Higher Orders of Stability and Efficiency
}

Chakrabarti, S., \& Tangsangasaksri, S. (2014). Network Topology, Higher Orders of Stability and Efficiency. International Game Theory Review, 16(4), [1450010]. https://doi.org/10.1142/S0219198914500108

\author{
Published in: \\ International Game Theory Review
}

Document Version:

Peer reviewed version

Queen's University Belfast - Research Portal:

Link to publication record in Queen's University Belfast Research Portal

Publisher rights

Copyright 2014 World Scientific Publishing Company.

This work is made available online in accordance with the publisher's policies. Please refer to any applicable terms of use of the publisher.

\section{General rights}

Copyright for the publications made accessible via the Queen's University Belfast Research Portal is retained by the author(s) and / or other copyright owners and it is a condition of accessing these publications that users recognise and abide by the legal requirements associated with these rights.

Take down policy

The Research Portal is Queen's institutional repository that provides access to Queen's research output. Every effort has been made to ensure that content in the Research Portal does not infringe any person's rights, or applicable UK laws. If you discover content in the Research Portal that you believe breaches copyright or violates any law, please contact openaccess@qub.ac.uk. 


\title{
Network Topology, Higher Orders of Stability and Efficiency*
}

\author{
Subhadip Chakrabarti ${ }^{\dagger} \&$ Supanit Tangsangasaksri ${ }^{\ddagger}$
}

January 6, 2014

\begin{abstract}
Stable networks of order $r$ where $r$ is a natural number refer to those networks that are immune to coalitional deviation of size $r$ or less. In this paper, we introduce stability of a finite order and examine its relation with efficient networks under anonymous and component additive value functions and the component-wise egalitarian allocation rule. In particular, we examine shapes of networks or network architectures that would resolve the conflict between stability and efficiency in the sense that if stable networks assume those shapes they would be efficient and if efficient networks assume those shapes, they would be stable with minimal further restrictions on value functions.
\end{abstract}

JEL Code: C62, C71.

Keywords: Stability of order $r$, Efficiency, Network architecture.

*We thank Matt Jackson, Robert Gilles and Sudipta Sarangi for their helpful suggestions. Thanks are also due to participants of the SAET Meetings 2005, Vigo, Spain and Bonn Graduate School Seminar Series for their comments. A previous draft of this paper entitled "Stable and Efficient Networks under Constrained Coalition Formation" was presented at the SAET meetings in Vigo in 2005. The paper was partly written when Subhadip Chakrabarti was a post doctoral research fellow at the University of Bonn. He gratefully acknowledges their hospitality and financial support.

†Queen's University Management School, Queen's University Belfast, Belfast, Northern Ireland, United Kingdom. E-mail: s.chakrabarti@qub.ac.uk

${ }^{\ddagger}$ Department of Economics, Thammasat University, Bangkok, Thailand. E-mail: supanitt@econ.tu.ac.th 


\section{Introduction}

The last few years have witnessed a rapid growth of papers devoted to social and economic networks. A subset of those papers have focussed on stability and efficiency and the conflict between them. Stable networks refer to those networks which once formed will be immune to further changes. The formation process itself is sometimes left unspecified. Efficient networks are those which maximize a social welfare function that is a sum of the utility or payoff functions of individuals. It is well documented there is a general conflict between stability and efficiency, namely, in many settings stable networks are not necessarily efficient and efficient networks are not necessarily stable.

The notion of stability is itself not uniquely defined but depend on what coordination possibilities are available to players or individuals. The existing models of network formation have focussed on two ends of a coordination spectrum. On one hand they have focussed on individual and pairwise solution concepts such as pairwise stability (see Jackson and Wolinsky (1996)) and strong pairwise stability (see Chakrabarti and Gilles (2007)). On the other hand, they have assumed complete coordination with the result being any arbitrary coalition including the grand coalition can freely form and members can alter the structure of links in the coalition (see Jackson and van den Nouweland (2005)).

In the real world very often, we can get intermediate levels of coordination resulting in formation of coalitions which are relatively small (smaller than the grand coalition) but at the same time bigger than a coalition of 1 . Therefore, from both a mathematical point of view as well as an economic one, such coalition formation is of interest and this is the subject of the present paper. Stable networks of order $r$ where $r$ is a natural number refer to those networks that are immune to coalitional deviation of size $r$ or less. In this paper, we introduce stability of a finite order and examine its relation with efficient networks.

The main objective is to identify specific classes of value functions where the conflict between stability of a certain order and efficiency is resolved. The focus of this paper is on the architecture based resolution of the conflict, namely, we investigate shapes of networks such that if efficient networks take those shapes, they are necessarily stable, and if stable networks take those shapes, they are necessarily efficient. 
The question of network topologies or shapes or architectures for which there is no conflict between stability and efficiency may be significant. If there are a vast number of such networks and one is looking for networks which are simultaneously stable and efficient, one may first focus on those topologies for which there is no conflict between stability and efficiency under reasonable assumptions.

We shall pursue the transferable utility framework introduced by Jackson and Wolinsky (1996). Under this framework, the network produces a certain value as defined by a value function which in turn is allocated among the players based on an allocation rule which determines the payoffs of the players. An example of such a framework would be, for instance, airline code-sharing, where the passenger embarking on a long distance flight pays an up-front fee which is distributed among airlines participating in the code-sharing network.

We assume that the value function follows certain well-known properties like anonymity and component additivity (see below for formal definitions) and focus on a certain allocation rule that has been popular in the literature, namely, the component-wise egalitarian allocation rule. Anonymity implies that value function only depends on the shape of the network and not the labels of the players. A component is a subnetwork where there is a path between any two players and no path from a player within the component to a player outside the component. Component additivity assumes away externalities across components. The component-wise egalitarian allocation rule divides the value produced by a component equally across the members of a component. These assumptions are undoubtedly strong and may not hold in real world settings but may serve as a starting point before we analyze more complicated networks.

The rest of the paper proceeds as follows. Section 2 introduces the notation and terminology. In Section 3 we impose the assumption of component additivity on value functions and under the component-wise egalitarian allocation rule, we look at that the configuration of efficiency compatible network architectures, by which we mean network shapes which ensure that if efficient networks assume those shapes, they are necessarily stable with no further restrictions on value functions. In Section 4, we impose the additional assumption of anonymity and we look at network architectures under which the conflict between stability of a certain order and efficiency is resolved. 
We find a broad class of architectures for which stable networks of a certain order are necessarily efficient subject to existence of both stable and efficient networks which assume those architectures. Section 5 concludes.

\section{Modelling Principles}

\subsection{Networks}

In this section we define the formal elements to describe network formation along with some concepts borrowed from graph theory. Let $N=\{1,2, \ldots, n\}$ be a finite set of players. A coalition refers to any subset of the player set and the size of a coalition is the number of players in that coalition. Two distinct players $i, j \in N$ with $i \neq j$ are linked if $i$ and $j$ are related in some capacity. Usually we think of such links as economically productive relationships between players. These relationships are undirected in the sense that the two players forming a relationship are equals within that relationship. We do not rule out that these relationships have spillover effects on the productive relations between other players.

Formally, an (undirected) link between $i$ and $j$ is defined as the set $\{i, j\}$. Throughout we use the shorthand notation $i j$ to denote the link $\{i, j\}$. It should be clear that $i j$ is completely equivalent to $j i$.

In total there are $\frac{1}{2} n(n-1)$ potential links on the player set $N$. The collection of these potential links on $N$ is denoted by

$$
g_{N}=\{i j \mid i, j \in N \text { and } i \neq j\}
$$

A network $g$ is now defined as any collection of links $g \subset g_{N}$. The collection of all networks on $N$ is denoted by $\mathbb{G}^{N}=\left\{g \mid g \subset g_{N}\right\}$. The collection $\mathbb{G}^{N}$ consists of $2^{\frac{1}{2} n(n-1)}$ networks. The network $g_{N}$ consisting of all links is called the complete network on $N$ and the network $g_{0}=\varnothing$ consisting of no links is the empty network on $N$.

Let $\pi: N \rightarrow N$ be a permutation on $N$. For every network $g \in \mathbb{G}^{N}$ the corresponding permutation is denoted by $g^{\pi}=\{\pi(i) \pi(j) \mid i j \in g\} \in \mathbb{G}^{N}$. Two networks $g, h \in \mathbb{G}^{N}$ have the same topology or architecture if there exists a permutation 
$\pi: N \rightarrow N$ such that $h=g^{\pi}$. This is denoted as $g \sim h$. For $g \in \mathbb{G}^{N}$ the corresponding network topology is denoted by $\bar{g}=\left\{h \in \mathbb{G}^{N} \mid h \sim g\right\}$. Clearly a network topology is a mathematical equivalence class with regard to the binary relationship $\sim$. It is obvious that the collection of all networks $\mathbb{G}^{N}$ is partitioned into network topologies.

For every network $g \in \mathbb{G}^{N}$ and every player $i \in N$ we denote $i$ 's neighborhood in $g$ by $N_{i}(g)=\{j \in N \mid j \neq i$ and $i j \in g\}$. Player $i$ therefore is participating in the links in her link set $L_{i}(g)=\left\{i j \in g \mid j \in N_{i}(g)\right\} \subset g$. We also define $N(g)=\cup_{i \in N} N_{i}(g)$ and let $n(g)=\# N(g)$ with the convention that if $N(g)=\varnothing$, we let $n(g)=1 .{ }^{1}$ Also, $n_{i}(g)=\# N_{i}(g) . n(g)$ will be referred to as size of the network $g$.

A path in $g$ connecting $i$ and $j$ is a set of distinct players $\left\{i_{1}, i_{2}, \ldots, i_{p}\right\} \subset N(g)$ with $p \geqslant 2$ such that $i_{1}=i, i_{p}=j$, and $\left\{i_{1} i_{2}, i_{2} i_{3}, \ldots, i_{p-1} i_{p}\right\} \subset g$. We say $i$ and $j$ are connected to each other if a path exists between them and they are disconnected otherwise. The network $g^{\prime} \subset g$ is a component of $g$ if for all $i \in N\left(g^{\prime}\right)$ and $j \in N\left(g^{\prime}\right)$, $i \neq j$, there exists a path in $g^{\prime}$ connecting $i$ and $j$ and for any $i \in N\left(g^{\prime}\right)$ and $j \in N(g)$, $i j \in g$ implies $i j \in g^{\prime}$. In other words, a component is simply a maximally connected subnetwork of $g$. We denote the set of network components of the network $g$ by $C(g)$. For any component $h \in C(g)$, the cardinality of $N(h)$ is the size of $h$.

The set of players that are not connected in the network $g$ are collected in the set of (fully) disconnected players in $g$ denoted by

$$
N_{0}(g)=N \backslash N(g)=\left\{i \in N \mid N_{i}(g)=\varnothing\right\} .
$$

Such players are also known as singletons. Furthermore, we define

$$
\Gamma(g)=\{N(h) \mid h \in C(g)\} \cup\left\{\{i\} \mid i \in N_{0}(g)\right\}
$$

as the partitioning of the player set $N$ based on the component structure of the network $g$. For any $g \in \mathbb{G}^{N}, S \in \Gamma(g)$, let $g(S)=g \cap g_{S}$ where $g_{S}=\{i j \mid i, j \in S \subset N$ and $i \neq j\}$. Namely, $g(S)$ denotes the subgraph of $g$ on the player set $S$.

Sometimes, depending on the context, we refer to a singleton as a null component, in which case a component as defined above is called a non-null component.

\footnotetext{
${ }^{1}$ We emphasize here that if $N(g) \neq \varnothing$, we have that $n(g) \geqslant 2$. Namely, in those cases the network has to consist of at least one link.
} 
We denote by $C^{r}(g)$ be the set of all non-null components of size less than or equal to $r$. A connected network is one where each pair of players are connected to each other. In other words, a connected network has one non-null component and no singletons. A network that is not connected is disconnected.

We shall emphasize certain network topologies. A star network is a connected network that has all players directly linked to a central player and no two other players directly linked to each other. A circle is a connected network in which every player is directly linked to two other players.

A critical player refers to a player who can by deleting a well chosen subset of links in her neighborhood link set can disconnect at least two other players. A network that has no critical players is referred to as a bi-connected network. ${ }^{2}$ A circle, for instance, is a bi-connected network.

\subsection{Value and Allocation}

The value function given by $v: \mathbb{G}^{N} \rightarrow \mathbb{R}$ such that $v(\varnothing)=0$ expresses the collective network benefits stemming from a certain network. ${ }^{3}$ A network value function $v$ assigns a total benefit $v(g) \in \mathbb{R}$ to the network $g \in \mathbb{G}^{N}$. The space of all network value functions $v$ such that $v(\varnothing)=0$ is denoted by $\mathbb{V}^{N}$. It is clear that $\mathbb{V}^{N}$ is a $\left(2^{\frac{1}{2} n(n-1)}-1\right)$-dimensional Euclidean vector space. The allocated payoff to an individual player is determined by an allocation rule $Y: \mathbb{G}^{N} \times \mathbb{V}^{N} \rightarrow \mathbb{R}^{N}$ which determines how the collective value is distributed over the individual players. $Y_{i}(g, v)$ is the payoff to player $i$ from the network $g$ under the value function $v$.

Let $v \in \mathbb{V}^{N}$ be some network value function. We consider two fundamental properties of such a network value function:

- The network value function $v$ is component additive if $v(g)=\sum_{h \in C(g)} v(h)$. Component additivity immediately implies that disconnected players $i \in N_{0}(g)$ generate no value.

\footnotetext{
${ }^{2}$ This is a standard graph theoretic term. Gilles et. al. (2006) refer to these as well-connected networks.

${ }^{3}$ The notation and terminology has been borrowed from Jackson and Wolinsky (1996).
} 
- The network value function $v$ is anonymous if $v\left(g^{\pi}\right)=v(g)$ for all permutations $\pi$ and networks $g$. Anonymity implies that benefits $v(g)$ depend on the topology of the network $g$ only.

Next we define some properties of an allocation rule. Recall that $\pi: N \rightarrow N$ is a permutation. Let $v^{\pi}$ be defined by $v^{\pi}\left(g^{\pi}\right)=v(g)$.

- An allocation rule $Y$ is anonymous if for any permutation $\pi, Y_{\pi(i)}\left(g^{\pi}, v^{\pi}\right)=$ $Y_{i}(g, v)$. Anonymity of the allocation rule simply means payoff of a player depends solely on the position in the network rather than the label of the players.

- An allocation rule $Y$ is balanced if $\sum_{i \in N} Y_{i}(g, v)=v(g)$ for all $v$ and $g$. Balancedness is a minimal property that will be assumed throughout.

- An allocation rule $Y$ is component balanced if $\sum_{i \in N} Y_{i}(h, v)=v(h)$ for every $g$ and $h \in C(g)$ and every component additive $v$. It is obvious that component balance implies balance. Component balance along with component additivity implies that fully disconnected players in $N_{0}(g)$ always have an allocated payoff of zero.

- An allocation rule is component decomposable if $Y_{i}(g, v)=Y_{i}(h, v)$ for all component additive $v \in \mathbb{V}^{N}, g \in \mathbb{G}^{N}, h \in C(g)$ and $i \in N(h)$. Component decomposability requires that if $v$ is component additive, the way that value is allocated within a component does not depend on the structure of other components.

Let $v \in \mathbb{V}^{N}$ be component additive. The component-wise egalitarian allocation rule is defined by

$$
Y_{i}^{c e}(g, v)=\frac{v\left(h_{i}\right)}{n\left(h_{i}\right)}
$$


where $h_{i} \in C(g)$ such that $i \in N\left(h_{i}\right)$ and $Y_{i}^{c e}(g, v)=\varnothing$ if there is no $h \in C(g)$ such that $i \in N(h)$. Under this rule, the value generated by a component is split equally among the members of that component. The component-wise egalitarian allocation rule satisfies anonymity, component balance and component decomposability.

Also, we consider the egalitarian allocation rule defined by

$$
Y_{i}^{e}(g, v)=\frac{v(g)}{n} \text { for all } i \in N .
$$

Clearly this allocation rule satisfies anonymity and balance, but not component balance or component decomposability. In our discussion these two allocation rules are featured prominently.

\subsection{Stability and Efficiency}

In this section we discuss network formation principles from a link-based perspective. Central to this approach is that the formation of a link in principle is considered separately. Each link in the network involves a pair of players. While mutual consent is required for establishing a link, each player can delete a link unilaterally.

Denote by $g+i j$, the network obtained by adding link $i j$ to the existing network $g$, i.e., $g+i j=g \cup\{i, j\}$. Similarly, $g-i j$ denotes the network that results from deleting link $i j$ from the existing network $g$, i.e., $g-i j=g \backslash\{i j\}$.

Similarly, denote by $g+h$, the network obtained by adding the link-set $h$ to the existing network $g$ where $h \subset g^{N} \backslash g$. For $h \subset g, g-h$ denotes the network that results by deleting the link-set $h$ from the existing network $g$.

We introduce three fundamental link formation principles.

- A network $g \in \mathbb{G}^{N}$ is link deletion proof (LDP) if for every player $i \in N$ and every neighbor $j \in N_{i}(g)$, it holds that $Y_{i}(g-i j, v) \leqslant Y_{i}(g)$. Link deletion proofness requires that each individual player has no incentive to sever an existing link with one of his neighbors.

- A network $g \in \mathbb{G}^{N}$ is strong link deletion proof (SLDP) if for every player $i \in N$ and every link-set $h \subset L_{i}(g)$, it holds that $Y_{i}(g-h, v) \leqslant Y_{i}(g, v)$. Strong link 
deletion proofness requires that each player has no incentive to sever links with one or more of his neighbors. Obviously, SLDP implies LDP.

- A network $g \in \mathbb{G}^{N}$ is link addition proof if for all players $i, j \in N$, it holds that $Y_{i}(g+i j, v)>Y_{i}(g, v)$ implies $Y_{j}(g+i j, v)<Y_{j}(g, v)$. Link addition proofness states that there are no incentives to form additional links.

These three fundamental stability concepts can be used to define additional stability concepts. A network $g \in \mathbb{G}^{N}$ is pairwise stable if it is link deletion proof and link addition proof. Furthermore, a network $g \in \mathbb{G}^{N}$ is strongly pairwise stable if it is strong link deletion proof and link addition proof.

Next we define certain notions of coalitional stability borrowed from Jackson and van den Nouweland (2005).

Let $S \subset N$ be an arbitrary coalition.

- A network $g^{\prime} \in \mathbb{G}^{N}$ is obtainable from $g \in \mathbb{G}^{N}$ via link deletion by $S$ if $g^{\prime} \subset g$ and $i j \in g$ and $i j \notin g^{\prime}$ implies $\{i, j\} \cap S \neq \varnothing$.

- A network $g^{\prime} \in \mathbb{G}^{N}$ is obtainable from $g \in \mathbb{G}^{N}$ via link addition by $S$ if $g^{\prime} \supset g$ and $i j \notin g$ and $i j \in g^{\prime}$ implies $\{i, j\} \subset S$.

These definitions reflect the fact that players can delete their links unilaterally but link addition requires cooperation of both players in question. Of course, coalitions can engage simultaneously in link addition and link deletion which we refer to as deviations.

- A network $g^{\prime} \in \mathbb{G}^{N}$ is obtainable from $g \in \mathbb{G}^{N}$ via deviations by $S$ if (i) ij $\in g$ and $i j \notin g^{\prime}$ implies $i j \cap S \neq \varnothing$.(ii) $i j \notin g$ and $i j \in g^{\prime}$ implies $i j \subset S$.

- A deviation by $S \subset N$ is profitable if there exists a network $g^{\prime}$ that is obtainable from $g$ by $S$ via deviation satisfying two properties: 
$-Y_{i}\left(g^{\prime}, v\right) \geqslant Y_{i}(g, v)$ for all $i \in S$.

- There exists $j \in S$ such that $Y_{j}\left(g^{\prime}, v\right)>Y_{j}(g, v)$.

Stable networks of order $r$ are those that are immune to profitable deviations by any coalition of size $r$ or less.

- A network $g$ is link deletion proof (link addition proof) of order $r \in \mathbb{N}$ (where $1 \leqslant r \leqslant n$ ) with respect to allocation rule $Y$ and value function $v$ if for any $S \subset N$ with $|S| \leqslant r, g^{\prime}$ that is obtainable from $g$ via link deletion (link addition) by $S$, and $i \in S$ such that $Y_{i}\left(g^{\prime}, v\right)>Y_{i}(g, v)$, there exists $j \in S$ such that $Y_{j}\left(g^{\prime}, v\right)<Y_{j}(g, v)$.

- A network $g$ is stable of order $r \in \mathbb{N}$ (where $1 \leqslant r \leqslant n$ ) with respect to allocation rule $Y$ and value function $v$ if for any $S \subset N$ with $|S| \leqslant r, g^{\prime}$ that is obtainable from $g$ via deviations by $S$, and $i \in S$ such that $Y_{i}\left(g^{\prime}, v\right)>Y_{i}(g, v)$, there exists $j \in S$ such that $Y_{j}\left(g^{\prime}, v\right)<Y_{j}(g, v)$.

Pairwise stability was seminally introduced by Jackson and Wolinsky (1996). Strong pairwise stability as defined above has been introduced by Gilles and Sarangi (2004). A similar concept has been introduced independently by Goyal and Joshi (2006) as "pairwise Nash equilibrium". Pairwise Nash equilibria are equivalent to strong pairwise stability. Strong stability as defined by Jackson and van den Nouweland (2005) is equivalent to stability of order $n$. Any notion of stability of order $r$ is stronger than the corresponding notion of order $r-1$ where $r \geqslant 2$. Stability of order 1 is equivalent to SLDP. Stability of order 2 is equivalent to "pairwise strong Nash equilibrium" as defined by Bloch and Bellafleme (2004).

It is important to note that stability of any order is stronger than link addition proofness and link deletion proofness of that order combined. In other words, a network may be both link addition proof and link deletion proof of order $r$ but not strong stable of order $r$. This is because it may be immune to both link formation and link deletion but not both simultaneously.

Finally, we define efficiency. 
- A network $g \in \mathbb{G}^{N}$ is efficient with respect to value function $v$ if $v(g) \geqslant v\left(g^{\prime}\right)$ for all $g^{\prime} \subset g_{N}$.

\subsection{Cooperative Games and the r-Core}

A $T U$ cooperative game is a pair $(N, w)$ where $N$ is the set of players, and $w: 2^{N} \rightarrow \mathbb{R}$ defines the productive value of a coalition, and is called the characteristic function. Since the player set is fixed, we will simply refer to the characteristic function as a cooperative game.

An allocation $x \in \mathbb{R}^{N}$ is in the $r$-core, where $r$ is a natural number less than or equal to $n$, if $\sum_{i \in N} x_{i}=w(N)$ and $\sum_{i \in S} x_{i} \geqslant w(S)$ for all $S \subset N$ such that $|S| \leqslant r$. The $\mathrm{n}$-core is simply referred to as the core. Obviously, any allocation in the $r$-core also belongs to the $(r-1)$-core.

For the purposes of this paper, we are interested in a special type of cooperative game, which we denote by $w^{v}$. For all $S \subset N$,

$$
w^{v}(S)=\max _{g \subset g_{S}} v(g) .
$$

Thus the value of a coalition is the maximum value that the coalition can obtain by rearranging its members in a network. The anonymity and component additivity of $v$ implies symmetry and additivity of $w^{v}$.

\section{Component Additive Value Functions}

\subsection{Deviation Proof Network Topologies}

We will begin by extending the analysis of Gilles et. al. (2006) who show that under component additive (but not necessarily anonymous) value functions, once efficient networks take certain shapes (namely, bi-connected graphs), then under $Y^{c e}$, all efficient networks are strongly pairwise stable without any further restrictions on value functions. This raises the possibility that appropriate architectures can resolve the conflict between stability and efficiency for higher orders of stability. Namely for stronger notions of stability, there is a wide class of architectures such that if efficient networks assume those architectures, they are stable without further restrictions on 
value functions. Unfortunately, this is not the case and architecture based resolution of the conflict cannot be extended to higher orders of stability.

We shall assume only component additivity of value functions in this section but not anonymity. We shall also restrict ourselves to the component-wise egalitarian allocation rule. We begin with the definition of inclusive networks.

Definition 1 A network $g^{\prime}$ which is obtainable from $g$ by deviation by a coalition $S \subset N$ is said to be inclusive of $g$ if for any $i, j \in N(h), h \in C(g)$, there exists $h^{\prime} \in C\left(g^{\prime}\right)$ such that $i, j \in N\left(h^{\prime}\right)$.

In other words, the deviation would not result in breaking up existing components into smaller components though it allows the connecting of existing components to form larger components. So all types link addition is allowed while only those link deletions are allowed which do not destroy all existing path between any two players. In Figure 1, $g_{2}$ is inclusive of $g_{1}$ while $g_{3}$ is not because in $g_{3}$, there is no path between 4 and 5 which were previously connected.

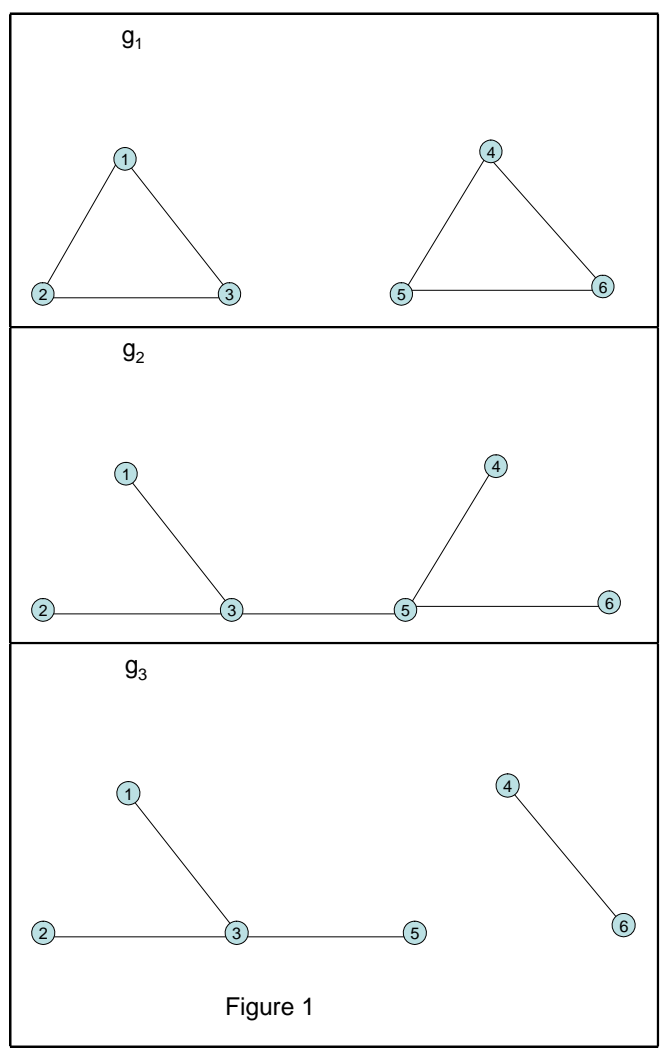


Next we have the following proposition.

Lemma 1 Let $v$ be component additive and $Y=Y^{c e}$. Given an efficient network $g$, there does not exist any profitable deviation that yields a network $g^{\prime}$ that is inclusive of $g$.

Proof. Towards a contradiction, suppose there exists a profitable deviation by an arbitrary coalition $S \subset N$ that yields a network $g^{\prime}$ that is inclusive with regard to $g$. We begin by showing that there can no player that can be strictly worse off as a result of such a deviation.

Consider any arbitrary player $i$ (which may or may not belong to the deviating coalition $S$ ). Let $h_{i}^{\prime}$ be a component (possibly a null component if $i$ is a singleton) containing $i$, namely, $i \in N\left(h_{i}^{\prime}\right)$ in the network $g^{\prime}$. Then there are two possibilities which we delineate as two cases.

Case 1: $N\left(h_{i}^{\prime}\right) \notin \Gamma(g)$.

Then, given that $g^{\prime}$ is inclusive of $g$, there exists a collection of player sets $A_{1}, A_{2}, \ldots, A_{m} \in \Gamma(g)$ such that

$$
N\left(h_{i}^{\prime}\right)=\bigcup_{l=1}^{m} A_{l}
$$

Two other conditions have to hold. First, $i$ must belong to one of the sets among $A_{1}, A_{2}, \ldots, A_{m}$. Call this set $A_{i}$. Second, $A_{i}$ must contain at least one member of the deviating coalition, namely, $A_{i} \cap S \neq \varnothing$. Let $j \in A_{i} \cap S$.

Since $j$ is a member of the deviating coalition, it must be the case that

$$
Y_{j}^{c e}\left(g^{\prime}, v\right) \geqslant Y_{j}^{c e}(g, v) .
$$

But, both $i$ and $j$ belong to $A_{i}$ and $N\left(h_{i}^{\prime}\right)$. Hence,

$$
\begin{aligned}
Y_{i}^{c e}(g, v) & =Y_{j}^{c e}(g, v) \\
Y_{i}^{c e}\left(g^{\prime}, v\right) & =Y_{j}^{c e}\left(g^{\prime}, v\right) .
\end{aligned}
$$

Hence,

$$
Y_{i}^{c e}\left(g^{\prime}, v\right) \geqslant Y_{i}^{c e}(g, v) .
$$

Case 2: $N\left(h_{i}^{\prime}\right) \in \Gamma(g)$. 
Then, there exists $h \in C(g) \cup N_{0}(g): N(h)=N\left(h_{i}^{\prime}\right)$. If $S \cap N\left(h_{i}^{\prime}\right)=\varnothing$, then $h_{i}^{\prime}=h$ and payoffs of $i$ remain unchanged from the definition of $Y^{c e}$. Next let $S \cap N\left(h_{i}^{\prime}\right) \neq \varnothing$ and let $j \in S \cap N\left(h_{i}^{\prime}\right)$.

But, both $i$ and $j$ belong to $N(h)$ and $N\left(h_{i}^{\prime}\right)$. Hence,

$$
\begin{aligned}
Y_{i}^{c e}(g, v) & =Y_{j}^{c e}(g, v) \\
Y_{i}^{c e}\left(g^{\prime}, v\right) & =Y_{j}^{c e}\left(g^{\prime}, v\right) .
\end{aligned}
$$

Since $j$ is a member of the deviating coalition, it must be the case that

$$
Y_{j}^{c e}\left(g^{\prime}, v\right) \geqslant Y_{j}^{c e}(g, v)
$$

From (8) to (10), we get

$$
Y_{i}^{c e}\left(g^{\prime}, v\right) \geqslant Y_{i}^{c e}(g, v) \text {. }
$$

Now, $i$ is a completely arbitrary player. Hence, it follows that in deviations leading to inclusive networks, there is no player in the player set $N$ that is worse off. But there is at least one player in the player set $S$ who is strictly better off from the definition of a profitable deviation. This contradicts that $g$ is efficient.

Proposition 1 immediately leads to the following corollary.

Corollary 1 Under any component additive $v$ and allocation rule $Y^{c e}$, an efficient network is link addition proof of any arbitrary order.

Proof. Link addition would only lead to inclusive networks. Hence, by Lemma 1, the result follows.

Hence, starting from an efficient network any profitable deviation must lead to a non-inclusive network. Next, we identify another type of deviation that is not profitable as well.

Definition 2 A player is said to isolate herself if she deletes all links in her neighborhood.

It is fairly simple of show that starting from an efficient network, isolation cannot be a profitable deviation. 
Lemma 2 Let $v$ be component additive and $Y=Y^{c e}$. Given any efficient network, there cannot be any profitable deviation involving a player isolating herself.

Proof. The empty network always yields a value of zero. So any component of an efficient network must yield a non-negative value. Because otherwise value can be increased by deleting all links in that component contradicting efficiency. Hence, each player gets a non-negative payoff. Isolation would always lead to a zero payoff. So isolation cannot be a profitable deviation.

By allowable deviations corresponding to a certain notion of stability, we mean the processes of link addition/link deletion by a coalition of appropriate size such that these processes do not yield profitable deviations for networks satisfying that notion of stability. For instance, when we are talking of strong pairwise stability, allowable deviations are link formation by coalitions of two players and link deletion by coalitions of one. With regard to strong stability, allowable deviation would include any conceivable deviation. Next, we can define deviation proof topologies.

Definition 3 A network architecture or topology is deviation proof with regard to some allowable deviation if it is not possible to form non-inclusive networks without isolation using such deviation.

Below in Table 1, we list deviation proof architectures for important types of allowable deviations. Next, we have the following proposition. It is an extension of Corollary 3.2 of Gilles et al. (2006).

Lemma 3 Let $v$ be component additive and $Y=Y^{c e}$. Then, if an efficient network has a deviation proof architecture, it is stable.

Proof. The only two things a coalition can do by deviating is either form an inclusive network or isolate themselves. By Lemma 1 and Lemma 2, neither is profitable.

Next, let us consider architectures such that if under a component additive value function, they turn out to be efficient, they must be stable as well. Let us call those architectures as efficiency compatible. From Lemma 3, deviation proof architectures are efficiency compatible. We show below that if a network is not deviation proof, we can find value functions for which it is not efficiency compatible. 


\begin{tabular}{|c|c|c|}
\hline Nature of Stability & $\begin{array}{l}\text { Nature of allowable } \\
\text { deviation }\end{array}$ & $\begin{array}{c}\text { All possible deviation } \\
\text { proof architectures }\end{array}$ \\
\hline SLDP & $\begin{array}{l}\text { Link deletion by } \\
\text { coalitions of size } 1\end{array}$ & Bi-connected networks \\
\hline Strong pairwise stability & $\begin{array}{l}\text { Link deletion by } \\
\text { coalitions of size } 1 \text {, } \\
\text { link formation by } \\
\text { coalitions of size } 2 \\
\end{array}$ & Bi-connected networks \\
\hline $\begin{array}{l}\text { Link addition proofness } \\
\text { of order } r \geqslant 2\end{array}$ & $\begin{array}{l}\text { Link addition } \\
\text { by arbitrary } \\
\text { coalitions of size } \\
2 \text { or more }\end{array}$ & All possible networks \\
\hline $\begin{array}{l}\text { Link deletion proofness } \\
\text { of order } r \geqslant 2\end{array}$ & $\begin{array}{l}\text { Link deletion } \\
\text { by coalitions of } \\
\text { size } 2 \text { or more }\end{array}$ & $\begin{array}{l}\text { Networks where } \\
\text { maximum size of each } \\
\text { non-null component } \\
\text { does not exceed } 2\end{array}$ \\
\hline Stability of order $r \geqslant 2$ & $\begin{array}{l}\text { Simultaneous link } \\
\text { addition and link } \\
\text { deletion by coalitions of } \\
\text { size } 2 \text { or more }\end{array}$ & Empty network \\
\hline
\end{tabular}

Table 1

Lemma 4 Let $v$ be component additive and $Y=Y^{c e}$. Given a network that is not deviation proof, we can find value functions for which it is not efficiency compatible.

Proof. Consider a network $g$ that has an architecture that is not deviation proof under the allowable deviation in question. We shall construct a value function under which $g$ may be efficient but not stable. Hence, we show that it is not efficiency compatible.

From Table 1, the allowable deviations must include link deletion because there does not exist a network that is not deviation proof under link addition alone.

First, suppose there exists a component $\widehat{h} \in C(g)$ such that $n(\widehat{h}) \geqslant 3$. Then 
there must exist a coalition $S \subset N(\widehat{h})$ where $|S| \geqslant 1$ that can form a smaller nonnull component $h^{\prime}$ by deleting links, namely $n\left(h^{\prime}\right)<n(\widehat{h})$ such that $S \subset N\left(h^{\prime}\right)$. This is obvious if allowable deviations include link deletion by coalitions of size 2 or more. If link deletion by coalitions of only size 1 are allowed, then, by Table 1, the network is not a bi-connected graph. Then take any critical player. By definition it can form a smaller component by deleting an appropriately chosen set of links in its neighborhood link-set.

Call the resulting network $g^{\prime}$. Assign a value 1 to $\widehat{h}$ and 0 to all other components, if any in $g$. Further, assign a value 1 to $h^{\prime}$ and a value 0 to all components other that $h^{\prime}$ that are formed by link deletion by $S$. Finally, assign values 0 to all other networks. Then, $g$ is an efficient network. But for all $j \in S$,

$$
Y_{j}\left(g^{\prime}, v\right)=\frac{1}{n\left(h^{\prime}\right)}>\frac{1}{n(\widehat{h})}=Y_{j}(\widehat{h}, v)=Y_{j}(g, v) .
$$

Therefore, $g$ is not stable.

Next consider a network $g$ where the size of the largest component does not exceed 2 and $g$ is not deviation proof with regard to the allowable deviation in question. We know from Table 1 that there must be at least one non-null component because the empty network is deviation proof for all possible allowable deviations. Further the only allowable deviation for which this is not deviation proof is simultaneous link formation and link deletion by coalitions of size 2 or more. Suppose, $n=3$. Then $g$ consists of a component of size 2 and a singleton, say $g=\{12\}$. Then assign a value 1 to the any components 12 and 23 and zero to all other components. Then 2 and 3 can induce a profitable deviation by 2 deleting its link with 1 and forming a link with 3. Hence, $g$ cannot be stable even though it is efficient. The same logic can be used to construct value functions under which any network with at least one singleton and one non-null component is efficient but not stable.

Finally consider a network $g$ where all components have size 2. There must necessarily be an even number of players. Consider an anonymous value function and assign a value 1 to components of size 2 or less and a value $\frac{7}{4}$ to a star component of size 3. Let all other components earn zero value. The network is efficient but not stable since a coalition of 2 can form a star and earn a higher payoff of $\frac{7}{12}>\frac{1}{2}$.

Lemma 4 is making a somewhat obvious point. A deviation proof architecture 
is efficiency compatible. A non-deviation proof architecture is not guaranteed to be efficiency compatible. The importance of the result lies in its negative implications. Deviation proof networks are few. So, the kind of analysis pioneered by Gilles et. al. (2006) cannot be extended to higher orders of stability.

\subsection{Communication Networks: An Example}

Communication networks were introduced by Myerson (1977) and subsequently elaborated upon, among others, by Owen (1986), Slikker and van den Nouweland (2001) and Jackson and van den Nouweland (2005). Let $z$ be a cooperative game. Hence, $z(S)$ indicates the productive value of an arbitrary coalition $S \subset N$.

Let us introduce the restriction that a coalition is productive only when all members of the coalition can communicate with each other. Communication can take place only along links in a communication network $g$ and each link in this network incurs a $\operatorname{cost} c$. This then allows one to define a value function that assigns to each network $g$ the productive value that the players can obtain when they have the communication lines in $g$ available, minus the cost of the network.

A given cooperative game $z$ and a cost per link $c$ lead to a value

$$
v^{z, c}=\sum_{S \in \Gamma(g)} z(S)-\sum_{i j \in g} c .
$$

In order to ensure that $v^{z, c}(\varnothing)=0$, we limit ourselves to zero-normalized characteristic functions, i.e. $z(\{i\})=0$ for each $i \in N$. Jackson and van den Nouweland (2005) show that if $z$ is convex and symmetric, then a strongly stable and efficient networks exists. We can do away with both convexity and symmetry but impose the restriction that $c=0$ and prove that there exists an efficient and strongly pairwise stable network. The reason is obvious. If $c=0$, value depends only on partitioning of the player set $\Gamma$ rather than the exact network structure. Therefore there exists a certain partition that yields the highest value. One can always find a bi-connected network that yields the efficient partition by replacing each component of an efficient network by a completely intra-connected component. Such a network will be both efficient and strongly pairwise stable. The same cannot be said of any order of stability equal to or higher than 2 . 
Lemma 5 For $v^{z, 0}$, there always exists a network that is both efficient and strongly pairwise stable.

\section{Component Size, Stability and Efficiency}

\subsection{Existence of Stable Networks and Efficiency}

In this section, we will explore the relationship between stability and efficiency conditional on the fact that one or the other exists but we shall make the additional rather strong assumption of anonymity. Under these two assumptions, namely, component additivity and anonymity of the value function and under the component-wise egalitarian allocation rule, Jackson and van den Nouweland (2005) have shown that the following facts are equivalent:

(i) A stable network of order $n$ exists;

(ii) The set of stable and efficient networks coincide;

(iii) The core of $w^{v}$ is non-empty.

We extend the results to stability of any arbitrary order. First, we start with some results that are not directly related to the question under investigation but will prove useful later on. The proof of the proposition below is a straightforward extension of Theorem 1 of Jackson and van den Nouweland (2005), and hence we skip the proof. ${ }^{4}$ Proposition 1 Consider any anonymous and component additive value function $v$. If $Y$ is an anonymous, component decomposable and component balanced allocation and $g \in \mathbb{G}^{N}$ is a network that is stable of order $r$ with respect to $v$ and $Y$, then the following would hold:

(a) If there is more than one component with size less than or equal to $r$, then all agents in those components earn identical payoffs.

(b) If there is any singleton, then all agents in components with size less than or equal to $r$ will earn zero payoffs.

(c) If there is any component with size less than or equal to $r$, any agent in a component of size greater than $r$ will earn payoffs that is greater than or equal to those of all agents in components of size less than or equal to $r$.

\footnotetext{
${ }^{4} \mathrm{~A}$ complete proof is however available in the working paper version of this paper available at http://mpra.ub.uni-muenchen.de/52749/4/MPRA_paper_52749.pdf.
} 
Next we introduce the notion of component-size which plays an important role in resolving the conflict between stability and efficiency. The component-size of a network is the size of the largest component in a network. Formally,

Definition 4 The component size of a network $g$ denoted by

$$
\vartheta(g)=\max _{S \in \Gamma(g)}|S|
$$

For stable networks of order $r$, whose component-size is less than or equal to $r$, the component wise egalitarian allocation rule coincides with the egalitarian allocation rule. If the network has more than one component, then it follows from Proposition 1 given that the component wise egalitarian allocation rule satisfies anonymity, component balance and component decomposability. If it has a single component, it is a immediate consequence of the component-wise egalitarian allocation rule. Next we have the following proposition which is a straightforward extension of Theorem 2 of Jackson and van den Nouweland (2005) and so we skip the proof. ${ }^{5}$

Proposition 2 (a) For a component additive and anonymous $v$ and allocation rule $Y^{c e}$, if there exists an efficient network of component-size less than or equal to $r$, then all stable networks of order $r$ with component-size less than or equal to $r$ (provided they exist) must be efficient.

(b) If there exists a network which is stable of order $r$ and has a component-size less than or equal to $r$, then the r-core of $w^{v}$ is non-empty.

The conditions imposed by Proposition 2(a) are rather stringent. There must exist both a stable network of component size less than equal to $r$ and an efficient network with component size less than or equal to $r$ in order to ensure that at least one stable network of order $r$ is efficient as well. However, we cannot strengthen the Proposition 2 in any way. In the working paper version of this paper, we give several counter-examples illustrating this. First, we show that if there does not exist an efficient network of component-size less than or equal to $r$, then stable networks of order $r$ are not necessarily efficient. Next, we show that if there exists efficient

\footnotetext{
${ }^{5} \mathrm{~A}$ complete proof are however available in the working paper version of this paper available at http://mpra.ub.uni-muenchen.de/52749/4/MPRA_paper_52749.pdf.
} 
networks of component size less than or equal to $r$, then stable networks of order $r$ with component size greater than $r$ are not necessarily efficient. Finally, we show that the existence of stable networks of order $r$ with component-size greater than $r$ does not guarantee that the $r$-core is non-empty.

Let us now consider the converse question. When are efficient networks stable of order $r$ ? First, it can be shown that an efficient network of component-size less than or equal to $r$ is not necessarily stable of order $r$ simply because a stable network of order $r$ may not exist. Can the problem be resolved by existence of a stable network of order $r$ ? Namely, if there exists a stable network of order $r$, are all efficient networks of component size less than equal to $r$ stable of order $r$. If $r=n$, Jackson and van den Nouweland (2005) have shown that this is the case and they utilize the core properties of the cooperative game $w^{v}$ (see above) in order to show that. However, their result cannot be extended in our context. Even if there exists a stable network of order $r$ with component-size less than or equal to $r$, and an efficient network with component size less than or equal to $r$, while it follows from Proposition 2(a) that the stable network must be efficient, the converse is not true. There can be efficient networks with component size less than or equal to $r$ which are not stable of order $r$. We provide a counter-example in the working paper version of our paper.

We will end this subsection with a comment on Jackson and van den Nouweland's (2005) Theorem 2. If efficient networks are not necessarily stable of order $r$ even when there exists stable networks of order $r$ with component-size less than or equal to $r$, why does the result hold for $r=n$ ? Below, we prove a result that directly leads to the aforesaid fact. We start with a lemma.

Lemma 6 Let $v$ be component additive and anonymous. If the $r$-core of $w^{v}$ is nonempty, then, the allocation $\widehat{x}$ defined by $\widehat{x}_{i}=\frac{w^{v}(N)}{n}$ belongs to the r-core.

The proof is a straightforward extension of the proof of Lemma 1 of Jackson and van den Nouweland (2005) and so we do not replicate it here. A proof is however available in the working paper version of this paper. Now, we can prove the following proposition.

Lemma 7 Let $v$ be component additive and anonymous. If the $r$-core of $w^{v}$ is nonempty, then starting from any efficient network with component-size less than or 
equal to $r$, there cannot be any profitable deviation that leads to another network with component-size less than or equal to $r$.

Proof. Let the network $g$ be efficient relative to $v$ and have a component-size less than or equal to $r$. Suppose the $r$-core of $w^{v}$ is non-empty. Then, from Lemma 6 , the allocation $\widehat{x}$ given by $\widehat{x}_{i}=\frac{w^{v}(N)}{n}$ belongs to the $r$-core. Then,

$$
\sum_{S \in \Gamma(g)} v(g(S))=v(g)=\sum_{i \in N} \widehat{x}_{i}
$$

Further, since the component-size of $g$ is less than or equal to $r$, from the definition of the $r$-core,

$$
\sum_{i \in S} \widehat{x}_{i} \geqslant w^{v}(S) \geqslant v(g(S)) \text { for each } S \in \Gamma(g) .
$$

Hence, all weak inequalities must hold with equality and therefore for each $S \in \Gamma(g)$,

$$
\sum_{i \in S} \widehat{x}_{i}=v(g(S))
$$

Now, given that under $\widehat{x}$, each player gets equal payoffs, namely, $\widehat{x}_{i}=\widehat{x}_{j}$ for each pair $i, j, i \neq j$, it implies

$$
Y_{i}^{c e}(g, v)=\widehat{x}_{i} \text { for each } i \in N \text {. }
$$

Now, consider any arbitrary network $g^{\prime}$ with component size less than or equal to $r$. From the definition of the $r$-core, it follows that

$$
\begin{aligned}
\sum_{i \in S} \widehat{x}_{i} & \geqslant w^{v}(S) \geqslant v\left(g^{\prime}(S)\right) \text { for each } S \in \Gamma\left(g^{\prime}\right) \\
& \Rightarrow \frac{1}{|S|} \sum_{i \in S} \widehat{x}_{i} \geqslant \frac{1}{|S|} \cdot v\left(g^{\prime}(S)\right) \\
& \Rightarrow \widehat{x}_{i} \geqslant Y_{i}^{c e}\left(g^{\prime}, v\right)
\end{aligned}
$$

since $\widehat{x}_{i}=\widehat{x}_{j}$ for each pair $i, j, i \neq j$.

From (11) and (12), it follows that

$$
Y_{i}^{c e}(g, v) \geqslant Y_{i}^{c e}\left(g^{\prime}, v\right)
$$

Therefore, the lemma follows.

We can combine Proposition 2(b) and Lemma 7 to get the following result. 
Proposition 3 Let $v$ be component additive and anonymous. If there exists a network which is stable of order $r$ and has a component-size less than or equal to $r$, then starting from any efficient network with component-size less than or equal to $r$, there cannot be any profitable deviation that leads to another network with component-size less than or equal to $r$.

Any arbitrary network has component-size less than or equal to $n$. So starting from a certain network, if there cannot be any profitable deviation leading to a network with component-size less than or equal to $n$, it simply means that network is strongly stable (or stable of order $n$ ). If $r=n$, Proposition 3 translates to the fact that, if there exists a strongly stable network, every efficient network is strongly stable. This is precisely part of Theorem 2 of Jackson and van den Nouweland (2005). Also, Lemma 7 implies that if the core of $w^{v}$ is non-empty, then every efficient network is strongly stable. Given that there always exists an efficient network, it implies that if the core of $w^{v}$ is non-empty, then there exists a strongly stable network.

Finally, note that the converse of Proposition 2(b) does not necessarily hold. Namely, the $r$-core can be non-empty but there need not exist any network that is stable of order $r$. To see this, reconsider the example below.

Example 1 Let $N=\{1,2, \ldots, 8\}$. Let $v$ be component additive and anonymous and a component which is a star network of size 4 yields a value 4 . Also, let any component with shape like that of $g_{2}$ in Figure 1 yield value 7 . Singletons yield zero value and so do all other network topologies. The efficient network is given by two star components of size 4 . But it is not stable of order 4 because the centers of stars can delete one link each, form a mutual link and earn a higher payoff of $7 / 6$ which is greater than their payoff in the efficient network, namely 1 . In fact, in this example there does not exist any stable network of order 4 . The allocation $x_{i}=1$ for all $i \in N$ belongs to the 4-core even though no stable networks exists.

We can summarize the results of this section as follows. For a given value function $v$ (which we assume is anonymous and component-additive), let $E(v)$ be the set of efficient networks and $S S^{r}(v, Y)$ be the set of stable networks of order $r$ under the allocation rule $Y$. Let

$$
\left.\mathbb{G}_{r}^{N}=\left\{g \mid C(g)=C^{r}(g)\right)\right\}=\{g \mid \vartheta(g) \leqslant r\}
$$


Hence, $\mathbb{G}_{r}^{N} \subset \mathbb{G}^{N}$ denotes the set of networks with component-size less than or equal to $r$.

Then,

$$
\begin{aligned}
E(v) \cap \mathbb{G}_{r}^{N} & \neq \varnothing \Rightarrow S S^{r}\left(v, Y^{c e}\right) \cap \mathbb{G}_{r}^{N} \subset E(v) ; \\
S S^{r}(v, Y) \cap \mathbb{G}_{r}^{N} & \neq \varnothing \Rightarrow Y_{i}^{c e}(g, v) \geqslant Y_{i}^{c e}\left(g^{\prime}, v\right) \text { for all } g \in E(v) \cap \mathbb{G}_{r}^{N}, g^{\prime} \in \mathbb{G}_{r}^{N} .
\end{aligned}
$$

\subsection{Convexity of Value Functions}

The next question we shall tackle is what restrictions on value functions guarantee the existence of at least one efficient network that is stable of order $r$.

From the above section, we have a sufficient condition:

(a) There exists at least one efficient network of component size less than or equal to $r$, and

(b) There exists of at least one stable network of component-size less than or equal to $r$.

We call it the $C S$ condition- $r$.

Jackson and van den Nouweland (2005) has given us another sufficient condition, namely, top-covexity. A value function is top-convex if

$$
\frac{w^{v}(N)}{n} \geqslant \frac{w^{v}(S)}{|S|}
$$

for all $S \subset N$.

Top convexity is both a necessary and sufficient condition for a strongly stable network to exist and under this condition, the set of stable networks coincide with the set of efficient networks. Given that there must always exist an efficient network, top-convexity guarantees the existence of an efficient and strongly stable network. It immediately follows that top convexity guarantees the existence of an efficient network which is stable of order $r$ for any arbitrary $r$.

CS Condition- $r$ is not necessarily weaker than top-convexity. Top-convexity guarantees existence of a stable network but not necessarily one with component-size less than $r$. In fact, neither condition implies the other. Value functions may satisfy top convexity but not CS condition- $r$. Also, value functions may also satisfy CS 
condition- $r$ but not top-convexity. When $r=n$ do the two conditions coincide. In the working paper version of our paper, we provide suitable counter-examples.

Top convexity is also necessary and sufficient for the core of $w^{v}$ to be non-empty. We can define a condition on value function (which is similar to top-convexity but weaker) which is necessary and sufficient for the existence of the $r$-core.

Definition 5 A value function is convex of order $r$ if

$$
\frac{w^{v}(N)}{n} \geqslant \frac{w^{v}(S)}{|S|}
$$

for all $S \subset N$ such that $|S| \leqslant r$.

Then we have the following lemma which is a straightforward extension of Jackson and van den Nouweland's (2005) Theorem 3 and so we skip the proof. ${ }^{6}$

Lemma 8 The $r$-core of $w^{v}$ is non-empty iff the value function is convex of order $r$.

Of course, convexity of order $r$ is a necessary (from Proposition 2(b)) but not sufficient condition for existence of a stable network of order $r$. In Example 1, for instance, the allocation of 1 to every player belongs to the 4-core where but there does not exist any network that is stable of order 4. To summarize:

(i) CS condition $r$ is a sufficient but not necessary condition for existence of an efficient network that is stable of order $r$.

(ii) Top convexity is a sufficient but not necessary condition for existence of an efficient network that is stable of order $r$.

(iii) Neither of the two above conditions implies the other but the two conditions coincide if $r=n$.

(iv) Convexity of order $r$ is necessary but not sufficient for existence of a stable network of order $r$.

\section{Conclusion}

In this paper we introduce stability of a finite order and examine its relationship with efficient networks. Stable networks of a finite order are those networks that are

\footnotetext{
${ }^{6} \mathrm{~A}$ complete proof are however available in the working paper version of this paper available at http://mpra.ub.uni-muenchen.de/52749/4/MPRA_paper_52749.pdf.
} 
immune to changes in terms of alterations in the existing structure of links when only coalition not exceeding a certain size can be formed. We focus in this paper on topologies or architectures of networks which if assumed by stable networks would ensure these are efficient and if assumed by efficient networks would ensure these are stable. Under component additivity of value functions and component-wise egalitarian allocation rule, there are no significant network architectures which if assumed by efficient networks ensures that they are stable of order $r$ (where $r \geqslant 2$ ) without further restrictions on value functions. This contrasts with the results of Gilles et al. (2006) who find a broad class of such architectures for strong pairwise stability, namely, bi-connected graphs. Once we add anonymity, it holds that if there exists at least one efficient network with component-size less than or equal to $r$, then all stable networks of order $r$ with component-size less than or equal to $r$ must be efficient. We show using counter-examples that the results cannot be strengthened in any way. From this result, we identify a set of sufficient conditions in terms of stable and efficient networks assuming certain architectures which we call CS condition- $r$ which ensures there exists a network that is stable of order $r$ and efficient. These condition coincides with top-convexity when $r$ is equal to the total number of players but otherwise neither follows from nor implies top convexity. 


\section{References}

[1] Bloch, F. and P. Bellafleme (2004) "Market Sharing Agreements and Collusive Networks", International Economic Review, 45 (2), 387-411.

[2] Chakrabarti, S. and R.P. Gilles (2007) "Network Potentials", Review of Economic Design, 11 (1), 13-52.

[3] Gilles, R.P., S. Chakrabarti, S. Sarangi and N. Badasyan (2006) "Critical Agents in Networks", Mathematical Social Sciences, 52 (3), 302-310.

[4] Gilles, R.P. and S. Sarangi (2004) "The Role of Trust in Costly Network Formation," Discussion Paper 2003-53, Tilburg University, Center for Economic Research.

[5] Goyal, S. and S. Joshi (2006) "Unequal Connections", International Journal of Game Theory, 34 (3), 319-349.

[6] Harary, F. (1995) Graph Theory, Addison Wesley, Oxford, U.K.

[7] Jackson, M. and A. Wolinsky (1996) "A Strategic Model of Social and Economic Networks", Journal of Economic Theory, 71 (1), 44-74.

[8] Jackson, M. and A. van den Nouweland (2005) "Strongly Stable Networks", Games and Economic Behavior, 51 (2), 420-444.

[9] Myerson, R.B., (1977) "Graphs and Cooperation in Games", Mathematics of Operations Research, 2(3) 225-229.

[10] Owen, G. (1986) "Value of Graph-Restricted Games", Siam Journal of Discrete Mathematics, 7(2), 210-221.

[11] Slikker, M. and A. van den Nouweland (2001) Social and Economic Networks in Cooperative Game Theory, Kluwer Academic Publishers, Boston, MA. 